\title{
The Analysis of the Effective Communication of Visual Information on Modern Exhibition Space
}

\author{
Yuan Chen ${ }^{1}$ \\ Shandong Women's College \\ Jinan, China \\ e-mail: zy_wlh@126.com
}

\author{
Xin Bian ${ }^{2}$ \\ Shandong Women's College \\ Jinan,China \\ e-mail:155051477@qq.com
}

\begin{abstract}
In recent years, the government has increased its support for cultural creative industry, which makes the exhibition play an important role in economic development as cultural "soft power". While effective communication of visual information will directly affect the success of exhibition. This thesis from the view of visual communication tries analyzing visual information content in exhibition and the factor affecting the effective communication of visual information and finally concludes the method of improving the efficiency of visual information transmission.
\end{abstract}

Keywords-exhibition; visual information; effective communication

\section{INTRODUCTION}

Exhibition as a comprehensive display media undertakes the function of information Display Space spreading and exchange. Early in primitive period, human society had already utilized simple display means or media to carry out demonstration activities. Nowadays, the exhibition has become "soft power" of local economic development. Exhibition display space as a media of information spreading is a dynamic process rather than a static process. Sponsors use various display means and technology of showing exhibits, shape atmosphere of display space and spread information to recommend the image of display body. This process reflects the nature of display_ information spreading and exchange. Exhibition is obviously different from the common display space. Specifically exhibition has distinct timeliness and seasonality, the general display time of the exhibition is $2-5$ days. Large amount of information is spread in short period which undoubtedly tests spectators' receptivity. How to improve the efficiency of visual information transmit in modern exhibition is a problem to ponder.

Information communication is a basic way to meet human material life and rich spiritual life. Only through the information communication, the need of human existence and development can be satisfied. Information language is mainly divided into the sensitive one and the rational one. In the sensitive one, people generate a fell and express it through graphic or visual language. While the rational one is a rational analysis. It is like the sensitive information language finally express through visual language. Thus we can obtain that effectively acquiring the visual Information is vital to the information spreading and communication.

\section{CONTENT OF VISUAL INFORMATION IN EXHIBITION}

Visual information in exhibition is various. Almost the elements we can see are all visual information. It includes the following aspects:

\section{A. Color}

In exhibition display space, the color mainly includes spatial interface color, exhibit color, publicity page color, lighting color and etc. Color as the direct visual element in display space affects location of spatial theme and selection of style and atmosphere. Color can let the display space obtain more emotionality artistry.(Fig.1)

\section{B. Image-text}

Image-text is the main mean to acquire the spreading information. Comparing with the color as visual feel, imagetext is the visual express. Image-text information in exhibition is reflected by logo design, single publicity page design, poster design, layout design and etc. (Fig.2, Fig.1)

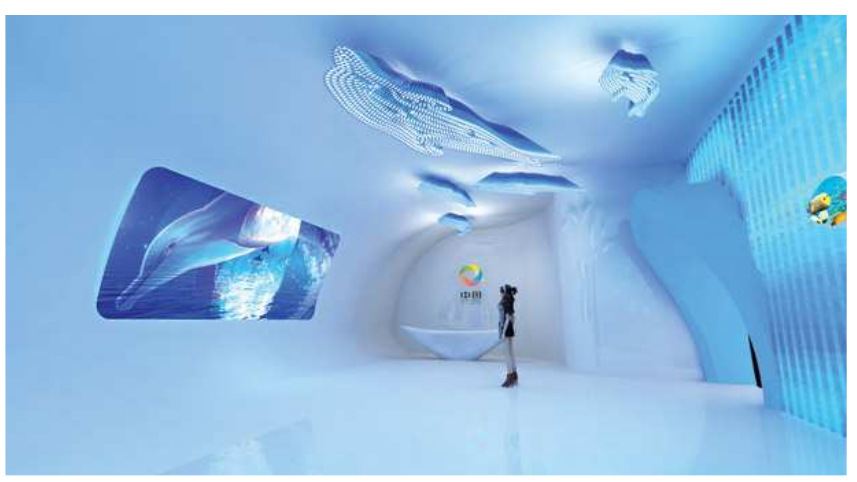

Fig. 1. The China Pavilion Of Yeosu Korea Expo 2012 


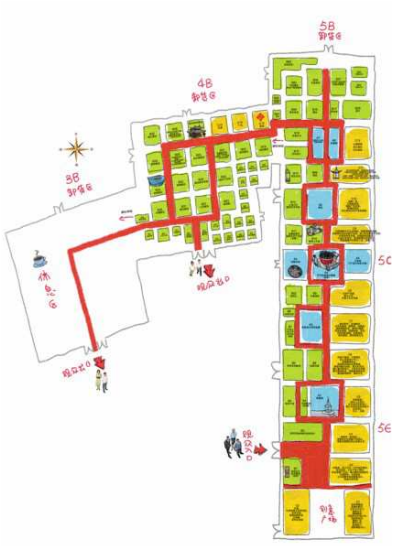

Fig. 2. The Map of China .Suzhou International Culture\&Design Industry Fair

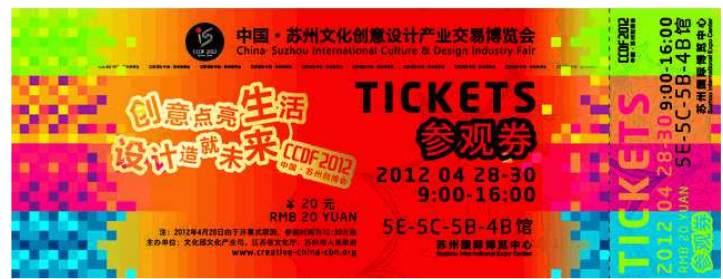

Fig. 3. The Tickets of the China.Suzhou International Culture\&Design Industry Fair

\section{Illumination}

Illumination design in exhibition display is mainly classified into space natural illumination and artificial illumination. There into artificial illumination includes integral illumination, local illumination, feature light. Illumination not only satisfies the need for indoor lighting, but also presents the display atmosphere. Because of the character of exhibition site, the art environment constructing by using natural illumination is far from enough as the basic illumination. It needs certain artificial illumination to control display effect and represent exhibit character.(Fig. 4)

\section{Material}

Material is a kind of visual information which is easily neglected. It is the necessary part of information express and constructing environment and also the basis for realizing the spatial creativity. Novel material and exquisite craftsmanship can attract spectators' attention to have a deep mental impression.(Fig. 5)

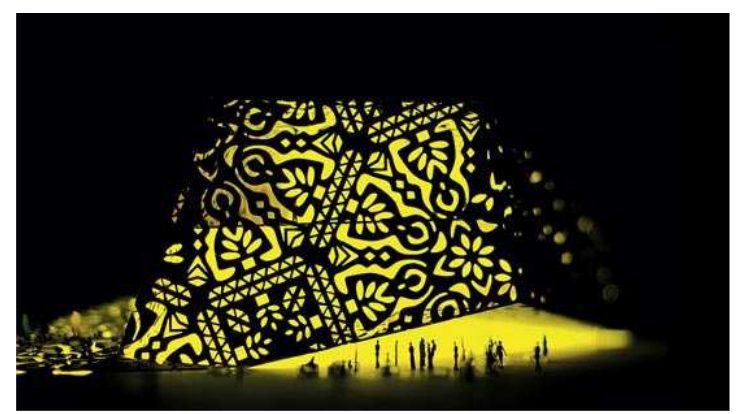

Fig. 4. The Poland Pavilion of Shanghai World Expo

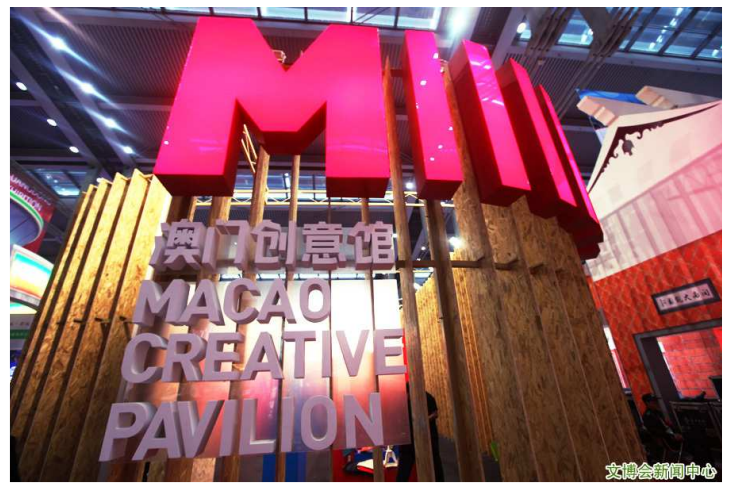

Fig. 5. The Macao Pavilion of the 8th ICCIE in Shenzhen

\section{THE FACTORS AFFECTING VISUAL INFORMATION TRANSMIT EFFICIENCY}

\section{A. Psychological factors}

Psychology is the reflection of objective things and their connections in brain. Analyzing information transmit efficiency besides considering the visual factors, it also needs to consider spectators psychological change. For example the different volume and scale of display space make the spectators have different cognitive effects. Large volume space generates a feel of greatness and broadness, and then the association that enterprise is powerful, has large investigation and good service. And the little space with the delicate exhibit and comfortable environment will give receiver such a psychological hint: high-end, taste, delicacy. The designer should according to receiver's psychological features design material space and emotional space. When the design idea and receiver's psychology conflict, the receiver's psychological demands should be respected and their action and interest of participating in the exhibition activities should be fully aroused. Thus, visual information transmission can gain the good effect.

\section{B. Visual factors}

Surveys shows that $83 \%$ human's cognition of surrounding environment and capture of information is gained by the visual. Eyes are like a camera, first shoot the thing, then introduce it into the brain, complete cognitive process. In exhibition display space, we cannot but consider visual information receptivity and The length of receiver's stay. Blindly pursue the enrichment and expand of information quantity of information transmit as many as possible in the extremely limited space, this action according to visual physiological law will greatly decrease the transmit efficiency. Firstly, the visual bearing scale is limited, lots of information crowding makes the spectators' visual uncomfortable and causes visual fatigue. While this inferior stimulation let them fell dull to the display things. Secondly, human's memory has oblivion rules. Numerous information cover is not useful for the memorization of main information. For example, a spectator visit the displace space which has too much to watch, carelessly watch all things and receives lots information. According to brain's oblivion rules, just a small part of the information which can be memorized and others are wasted. At last, complicated and strange visual image will occupy multidimensional information channel. When people are 
unfamiliar with the display image, their central nervous will occupy more information channels and the speed of cognition becomes slow. While using the simple image will make the spectator memorize easily and have deep impression. This is the simplicity of visual information. Therefore we should in order and efficiently have reasonable arrangement for transmitting information to improve the transit efficiency.

\section{Technical factors}

The technical level can directly or indirectly affects the efficiency of information transmission. With the development of scientific and the appearance of various new techniques and materials, exhibition as the display platform of new media and technology satisfies receivers' curiosity and desire for knowledge. Expression of multimedia technology expands exhibition capacity makes single and traditional static display transform into the multiple and dynamic display. For example, Shanghai World Expo set up the online World Expo to be convenient for spectators to visit(Fig. 6). It comprehensively introduced the World Expo through words, images, video and three-dimensional animation to let the spectators know dynamics of World Expo staying at home. Moreover Zaozhuang Pavilion of the 4th ICCIE in Shandong adopted virtual interactive equipment. Interactive video equipment is installed on the ground for audience entertainment and ornamental. It fully abstracts local cultural character and realizes the perfect combination of culture, technology and art.(Fig. 7)

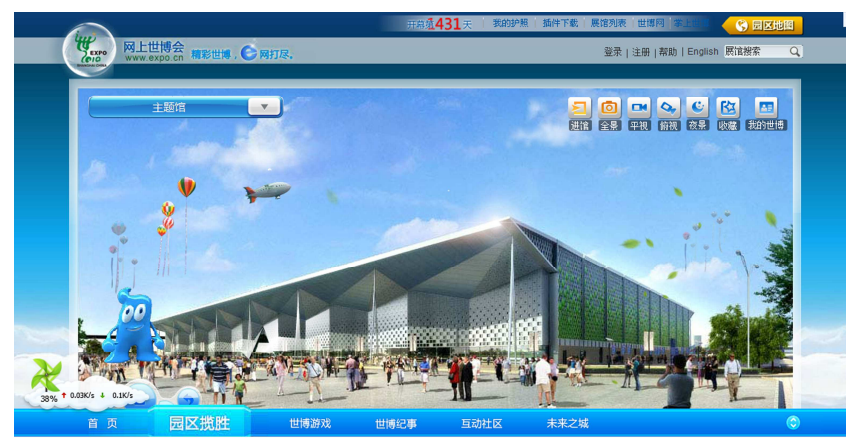

Figure 5. The online World Expo Of Shanghai World Expo

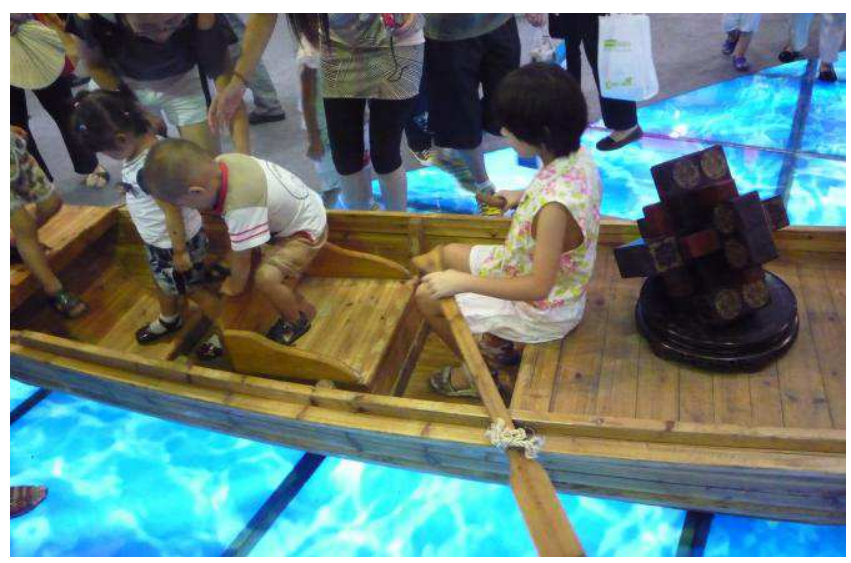

Figure 6. Zaozhuang Pavilion of the 4th ICCIE in Shandong

\section{METHODS OF IMPROVING VISUAL INFORMATION TRANSMIT EFFICIENCY}

Exhibition can efficiently spread information and is the window for numerous exhibitors to promulgate enterprise image and culture. To at maximum attract receiver's interest, at the aspects such as the display image, display method and display content exhibitors adopt various forms to compete with each other. But the multidimensional display information can not reflect the efficient receive of information. We should from the scientific view utilize the advantage of visual transmission and improve the information transmit efficiency to let the receivers digest the information in the shortest time. Only in this way the biggest benefit can be gained. Through the survey, we summarize the following methods to improve the efficiency of visual information transmission:

\section{A. Integrating information}

In actual life, what we contact with are isolated individuals, while these individuals connect with each other. To acquire precise and complete information, our brain is needed to screen and integrate the present sensitive materials and express by using corresponding media and technical method. In exhibition Display Space, the information transmission is such a process. The designers collect numerous relevant information select and handle the muddle materials and information. For example, in the initial design of the exhibition, designers should fully analyze the object the display information transmits to. And they should investigate receivers' age, sex, job and hobby and etc. Then choose the valuable information and rearrange them. The information integration can help us classify the primary and secondary and choose the suitable technical methods and display media to accurately design display content according spectators' interest. This can not only improve the efficiency but also the quality of information transmission.

\section{B. Controlling Information amount}

Vision is the main method for human to know the world and get information. Comparing with other methods of feeling, the information the vision gets is the most. However, the information receptivity is not equal to information storage. Contrarily the large information acquisition sometimes can affect information storage. To get the best transmit effect, information should be extracted and processed. Visual sign which increases the recognition speed is chosen in display activities as possible. At the same time, in the exhibition Display Space, clear information signs should be adopted as possible to reach the normal value of visual information capacity. For example, enlarge the area of visual sign; adopt contrast technique of large and small, sparseness and intense, Brightness and dinginess and etc. to improve the visual Cognition; Using modeling, color and other visual elements replaces literal introduction to shorten the fixation time and increase the information amount. While in the gangway, passage and other spatial environments where is not convenient for the spectators to stay, excess visual transmit information will not be arranged.

\section{Increasing information intensity}


The so-called increasing information intensity refers to letting the transmission strength of transmit information exceed other information's to exclude other secondary information. In exhibition display space, there are many ways to stress and intensify the information. For example, use the center set method to stress it in the location, then use special illumination to render the atmosphere and highlight to reach the high efficiency of information transmission through the method of emphasis; also can use illumination to highlight the enterprise name, enlarge sign or typeface, use special materials and methods to show the exhibits, still can weaken surrounding environment and secondary information to stress important information. In total, these methods of increasing information intensity can let the display visual information more striking and attractive.

\section{Selecting the efficient transmit method}

Exhibition activity is a multi - directional opening activity of information spreading and exchange the information. The development of the modern technology extends many media methods and the way of receiving information becomes multidimensional and shortcut. It is possible for diverse transmit methods to interfere receivers the diverse transmit methods without effective guide. To lead the spectator to comprehensively know the information, firstly consider the content of exhibition information transmission from the outside to the inside and proceed from one point to another. For example, displaying a handicraft, the key point of display it is not purely place a product, moreover make the spectators have a process of receiving information from sensitive to rational recognition through the exhibits' price and constructing cultural atmosphere and etc. Then, make the exhibition method to express clear, exact and active information. Traditional and single static exhibition method can not meet spectators' psychology of requiring various change, modern exhibition need us to choose various display methods to comprehensively and actively grasp exhibits' character. At last use the flexible and reasonable display forms. If the limited time and space can not allow comprehensively showing the content of display, we can spread the print information materials to the spectators or host Special report, forum and use other forms to transmit information.

\section{ACKNOWLEDGMENT}

Vision is information window and transmission is information media. Visual information as the main transmit method in the exhibition display space relates to the spectators' information acquisition and the exhibitors' economic interests and exhibition object. Analysis shows that the efficient transmission of visual information has ways to be found. By the way of integrating information, controlling information amount, increasing information intensity and selecting the efficient transmit method, we can clearly and accurately transmit visual information accompanied by the improvement of transmission efficiency.

\section{REFERENCES}

[1] Luo Yue, Wang Lin,Wu Meng. "Display Conception and Design," Tianjin science and technology press.2004.

[2] Zhu Chun, "Display Design," Chinese Fine Arts Publishing House. April,2000.

[3] Li Zhongfa. "Design Information Science," Sichuang Art Press. Aug,2004.

[4] (USA) Jan Lorenc, (USA) Lee H. Sikenike, (Australia) Craig Berge.Zheng Hanyu, Zhang Wenyin, Zhu Biao (translat). "What is Exhibition Design?" China Youth Press, 2008.

[5] Cheng Yuan. "Chinese Modern Exhibition Visual Image Design Analysis_ - as a Example of the 4th Shangdong ICCIE," 2013 master degree theses of Shangdong University.

[6] Shan Dandan. "Analyzing the Visual Transmission Efficiency of Exhibition Design," 2011 master degree theses of Nanjing Forestry University.

[7] Zhang Yin. "Importance of visual transmission in display design, Beauty and Era," late issue Julu,2004.

[8] Li Jiang, "Display Design Color," Guangxi Art Press. 2005.

[9] Cai Tao. "Transmission Space Design," China Youth Press. 2006. 